\title{
Single Center Experiences with Pegfilgrastim as Secondary Prophylaxis
}

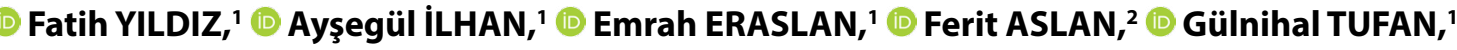 \\ Berna ÖKSÜZOĞLU, ${ }^{1}$ (1) Umut DEMIRCi ${ }^{3}$
}

\author{
'Department of Medical Oncology, University of Health Sciences, Dr. A.Y. Ankara Oncology Hospital, Ankara-Turkey \\ ${ }^{2}$ Department of Medical Oncology, Medical Park Hospital, Ankara-Turkey \\ ${ }^{3}$ Department of Medical Oncology, Memorial Hospital, Ankara-Turkey
}

\begin{abstract}
OBJECTIVE
Pegfilgrastim is a new generation on granulocyte-colony stimulating factors (G-CSF) with different pharmacokinetic properties. In this study, we aimed to evaluate the results of the use of pegfilgrastim as secondary prophylaxis.
\end{abstract}

\section{METHODS}

Between November 2016 and June 2019, a retrospective analysis of the patients who used pegfilgrastim with solid malignancies treated in the University of Health Sciences, Dr. A. Y. Ankara Oncology Hospital Department of Medical Oncology was performed.

\section{RESULTS}

A total of 148 patients were evaluated, and 33 (22.2\%) of them were in the geriatric population (age $>65)$. The majority of the patients $(n=97,65.5 \%)$ were diagnosed with breast carcinoma, and $93.2 \%(n=138)$ of all patients were treated with the intermediate-risk chemotherapy regimen. Pegfilgrastim was used as secondary prophylaxis for all of the patients. Despite pegfilgrastim prophylaxis, febrile neutropenia (FN) developed in four (2.7\%) patients. Chemotherapy delay due to afebrile neutropenia occurred in eight patients (5.4\%), and neutrophil counts returned to normal in mean 4.0 (3-5) days. When side effects were evaluated, 31 patients $(20.9 \%)$ had pegfilgrastim-induced bone pain and two patients $(1.3 \%)$ had redness in the injection site.

\section{CONCLUSION}

The low incidence of FN and chemotherapy delay, even in secondary prophylaxis with pegfilgrastim, suggests that pegfilgrastim is an effective agent in neutropenia prophylaxis.

Keywords: Febrile neutropenia; granulocyte colony-stimulating factors; pegfilgrastim.

Copyright ( 2020, Turkish Society for Radiation Oncology

\section{Introduction}

Although it may vary depending on the chemotherapy regimen, up to $80 \%$ neutropenia and $20-40 \%$ febrile neutropenia (FN) may occur due to cytotoxic chemotherapies.[1-4] Dose reduction and delaying due to neutropenia reduce the efficacy of chemotherapy. FN can be fatal and increases the costs of treatment due to hospitalizations and antibiotic use.[5,6]

The use of G-CSF significantly reduces the rates of neutropenia and the presence of FN due to chemotherapy. Filgrastim is a widely-used recombinant human 
G-CSF, which lasts longer than 25 years in the treatment of neutropenia. Pegfilgrastim is a new generation G-CSF with different pharmacokinetic properties. [5-8] Pegfilgrastim, which is formed by pegylation of filgrastim with polyethylene glycol, has less renal clearance due to its large molecular structure. In addition, pegfilgrastim binds to the surface receptors of neutrophil precursors and does not separate from the cell surface until the number of neutrophils increases. Due to these two properties, pegfilgrastim is long-acting. In clinical trials, pegfilgrastim administered as a single dose was compared with daily injections of filgrastim and similar efficacy on neutropenia and FN has been shown.[9-13]

In this report, we aimed to evaluate the results of the use of pegfilgrastim as secondary prophylaxis in patients who were diagnosed with solid malignancy and treated in our center.

\section{Materials and Methods}

Between November 2016 and June 2019, a retrospective analysis of the patients who used pegfilgrastim with solid malignancies treated at the University of Health Sciences, Dr. A. Y. Ankara Oncology Hospital Department of Medical Oncology, was performed.

Patients with solid malignancy, $>18$ years old and who used pegfilgrastim at least once for FN prophylaxis were included in this study. Patients with hematologic malignancies were excluded from this study. Due to the regulations in our country, all patients who received pegfilgrastim had a neutrophil count $<500$ or FN at least once after previous chemotherapy cycles.

Patients' demographic characteristics, comorbidity, Eastern Cooperative Oncology Group (ECOG) performance score, type and stage of the malignancy, chemotherapy regimen, previous radiotherapy, number of cycles the pegfilgrastim applied, chemotherapy delayed due to neutropenia, history of FN, hospitalization due to FN, duration of grade 4 neutropenia and pegfilgrastim related side effects were recorded.

Chemotherapy regimens were grouped according to the guidelines of the National Comprehensive Cancer Network (NCCN) 2.2018 for the risk of FN.

Neutropenia was defined as an absolute neutrophil count of $<1500 \mathrm{mcL}$. The fever $\left(\geq 38.3^{\circ} \mathrm{C}\right.$ or $38.0^{\circ} \mathrm{C}$ over 1 hour) with grade 4 neutropenia (neutrophil $<500$ $\mathrm{mcL}$ ) was accepted as FN.

Descriptive statistics were performed using the SPSS 23 statistical program.

\section{Results}

The clinical characteristics of the 148 patients included in this study are shown in Table 1. Thirty-three $(n=33,22.2 \%)$ patients were in the geriatric population (age $>65)$. The majority of patients had a diagnosis of breast carcinoma ( $\mathrm{n}=97,65.5 \%)$, and $92(62.2 \%)$ of all patients received adjuvant chemotherapy. Only one patient at the metastatic stage had bone marrow metastasis.

The most commonly used chemotherapy regimen was the combination of doxorubicin and cyclophosphamide $(n=81,54.7 \%)$. According to the risk of FN of the given chemotherapies, 10 patients $(6.7 \%)$ had high-risk chemotherapy, and the other patients received intermediate-risk chemotherapy. In our study, there was no patient who received neoadjuvant

\begin{tabular}{|c|c|c|}
\hline & $n=148$ & $\%$ \\
\hline \multicolumn{3}{|l|}{ Age } \\
\hline Median & $56(22-75)$ & \\
\hline \multicolumn{3}{|l|}{ Sex } \\
\hline Female & 120 & 81.1 \\
\hline Male & 28 & 18.9 \\
\hline \multicolumn{3}{|l|}{ Comorbidity } \\
\hline Yes & 67 & 45.3 \\
\hline No & 81 & 54.7 \\
\hline \multicolumn{3}{|l|}{ Performance score } \\
\hline 0 & 99 & 66.9 \\
\hline 1 & 45 & 30.4 \\
\hline 2 & 4 & 2.7 \\
\hline \multicolumn{3}{|l|}{ Malignancy } \\
\hline Breast & 97 & 65.5 \\
\hline Colorectal & 27 & 18.3 \\
\hline Lung & 5 & 3.4 \\
\hline Gynecologic & 5 & 3.4 \\
\hline Gastric & 4 & 2.7 \\
\hline Others & 10 & 6.7 \\
\hline \multicolumn{3}{|l|}{ Stage } \\
\hline Localized (Stage I-II-III) & 92 & 62.1 \\
\hline Stage IV & 56 & 37.8 \\
\hline \multicolumn{3}{|l|}{ Chemotherapy regimen } \\
\hline AC & 81 & 54.7 \\
\hline FOLFOX+/-biologic agents & 24 & 16.2 \\
\hline Docetaxel & 13 & 8.7 \\
\hline FOLFIRI+biologic agents & 8 & 5.4 \\
\hline Cisplatin+etoposid & 4 & 2.7 \\
\hline Others & 18 & 12.1 \\
\hline
\end{tabular}

AC: Doxorubicin/cyclophosphamide; FOLFOX: 5-Fluorouracil/leucovorin/ oxaliplatin; FOLFIRI: 5-Fluorouracil/leucovorin/Irinotecan 
chemotherapy or used pegfilgrastim simultaneously with radiotherapy.

A single dose of $6 \mathrm{mg}$ pegfilgrastim was administered subcutaneously 24 hours after chemotherapy, during median 3 (1-12), a totally of 425 chemotherapy cycles.

Despite pegfilgrastim prophylaxis, FN developed in four (2.7\%) patients. One patient was over 65 years of age, and the other patient had high-risk chemotherapy. All of these patients were hospitalized, and the mean duration of grade 4 neutropenia was two (1-3) days. No additional dose of G-CSF (filgrastim, lenograstim) was applied to patients after the development of FN.

One patient with metastatic breast carcinoma who had previously undergone multiple-line chemotherapy was died due to sepsis. This patient was being treated with eribulin at the sixth line for metastatic breast cancer. Before the third cycle of eribulin mesylate administration, the patient's ECOG performance score was 1, white blood cell (WBC) and neutrophil was 7500 $\mathrm{mL} / 3500 \mathrm{~mL}$, respectively. Although pegfilgrastim was administered 24 hours after chemotherapy, septic shock developed after 10 days.

Chemotherapy delay due to afebrile neutropenia occurred in eight patients (5.4\%), and neutrophil counts returned to normal in mean 4.0 (3.0-5.0) days.

When the side effects were evaluated, pegfilgrastiminduced bone pain was present in 31 patients (20.9\%), and redness in the injection site was 2 (1.3\%). Median 18.3 (3.5-30.5) months follow-up, no secondary malignancy occurred. There was no patient whose pegfilgrastim prophylaxis was stopped due to side effects.

\section{Discussion}

Despite advances in cancer treatment and prevention, neutropenia due to chemotherapy remains a common complication. Chemotherapy delays due to neutropenia may lead to a decrease in treatment efficacy, and FN can be fatal. There is a clear relationship between the intensity of the chemotherapy regimen and the risk of FN. In some regimens, the risk of FN may exceed $20 \%$. The use of G-CSF significantly reduces the risk of FN and prevents the chemotherapy delay due to neutropenia. In this way, the effectiveness of chemotherapy is preserved.[14] In this study, we aimed to evaluate the efficacy of new generation long-acting pegfilgrastim as secondary prophylaxis.

In this study, despite the pegfilgrastim prophylaxis, the incidence of development of FN was $2.7 \%$. In phase 3 , randomized trial, which compared pegfilgras- tim with filgrastim in patients with breast carcinoma, the incidence of $\mathrm{FN}$ was seen $13 \%$ in the pegfilgrastim arm after combination chemotherapy of docetaxel and doxorubicin.[9] In another study conducted in the similar group with a same chemotherapy regimen, the incidence of FN was reported as 7\%.[12] A study of 943 patients from nine European countries evaluated the efficacy of pegfilgrastim in high-risk chemotherapy.[15] The majority of the patients in this study were diagnosed with breast carcinoma (77\%), and the incidence of $\mathrm{FN}$ with pegfilgrastim prophylaxis was $3 \%$. A slightly higher incidence of FN was reported in these studies compared to our study. In our study 93.3\% of the patients were administered intermediaterisk chemotherapy regimen. In the other studies, all patients were administered a high-risk chemotherapy regimen. Thus, the $\mathrm{FN}$ rate is higher than the $\mathrm{FN}$ rate in our study.

A lower incidence of FN has been reported in patients receiving intermediate-risk chemotherapy with pegfilgrastim prophylaxis. In patients with colorectal cancer, the efficacy of pegfilgrastim after the 5-fluorouracil, leucovorin and oxaliplatin combination (FOLFOX)/5-fluorouracil, leucovorin and irinotecan combination (FOLFIRI) regimen was evaluated, and the $\mathrm{FN}$ rate was found 3.2\%.[16] In another study that compared placebo and pegfilgrastim after docetaxel in breast carcinoma, the ratio of $\mathrm{FN}$ was $17 \%$ and $1 \%$, respectively.[11] In these two studies, which were performed with intermediate-risk chemotherapy regimens, the FN rates were similar to our study.

In Vogel et al.'s study, the rate of hospitalization due to $\mathrm{FN}$ was reported as $1 \%$ in the pegfilgrastim arm. [11] In our study, all patients who developed FN were hospitalized. In our daily practice, the low socio-cultural level of our patients, and that they live in other cities make us more liberal in the decision of hospitalization, which might have led to a change in hospitalization rates.

In our study, the mean duration of grade 4 neutropenia was two (1-3) days. Similar to our study, Holmes et al. reported that the mean duration of grade 4 neutropenia for the patients who developed FN was 1.7 days.[12] In Salmon et al.'s study, chemotherapy delay due to neutropenia was also in $3 \%$ of the patients. [15] The incidence of FN and chemotherapy delay in this study is similar to our study.

In the previous studies, it was reported that the frequency of bone pain due to pegfilgrastim was $18-37 \%$. $[17,18]$ Similarly, these adverse effects were observed in $17.1 \%$ of our patients. 
One of the limitations of this study was the heterogeneous group of patients concerning oncologic diagnoses and chemotherapy regimens. The other limitation was the retrospective feature of our study.

Although all the patients in our study consisted of patients who had previously developed grade 4 neutropenia, it was observed that there was a low incidence of FN and chemotherapy delay in secondary prophylaxis with pegfilgrastim.

\section{Conclusion}

In this study, it was clearly seen that pegfilgrastim is an effective agent for neutropenia prophylaxis with a good tolerability profile.

Peer-review: Externally peer-reviewed.

Conflict of Interest: The authors have no conflicts of interest to declare.

Ethics Committee Approval: The authors declare that this research was conducted according to the principles of the World Medical Association Declaration of Helsinki "Ethical Principles for Medical Research Involving Human Subjects" (amended in October 2013).

Financial Support: The authors declare that this study received no financial support.

Authorship contributions: Concept - F.Y., U.D., B.Ö.; Design - F.Y., U.D.; Supervision - B.Ö., U.D.; Funding None; Materials - F.Y., A.İ., F.A.; Data collection and/or processing - F.Y., A.I.; Data analysis and/or interpretation F.Y., E.E., F.A.; Literature search - F.Y., G.T.; Writing - F.Y., G.T., E.E.; Critical review - F.Y., B.Ö., U.D.

\section{References}

1. Hashiguchi Y, Kasai M, Fukuda T, Ichimura T, Yasui T, Sumi T. Chemotherapy-induced neutropenia and febrile neutropenia in patients with gynecologic malignancy. Anticancer Drugs 2015;26(10):1054-60.

2. Green MD, Koelbl H, Baselga J, Galid A, Guillem V, Gascon $\mathrm{P}$, et al. A randomized double-blind multicenter phase III study of fixed-dose single-administration pegfilgrastim versus daily filgrastim in patients receiving myelosuppressive chemotherapy. Ann Oncol 2003;14(1):29-35.

3. Dinan MA, Hirsch BR, Lyman GH. Management of Chemotherapy-Induced Neutropenia: Measuring Quality, Cost, and Value. J Natl Compr Canc Netw 2015;13(1):e1-7.

4. Dale DC. Colony-stimulating factors for the management of neutropenia in cancer patients. Drugs 2002;62(Suppl 1):1-15.
5. Biganzoli L, Untch M, Skacel T, Pico JL. Neulasta (pegfilgrastim): a once-per-cycle option for the management of chemotherapy-induced neutropenia. Semin Oncol 2004;31(3 Suppl 8):27-34.

6. Almenar D, Mayans J, Juan O, Bueno JM, Lopez JI, Frau A, et al. Pegfilgrastim and daily granulocyte colony-stimulating factor: patterns of use and neutropenia-related outcomes in cancer patients in Spain-results of the LEARN Study. Eur J Cancer Care (Engl) 2009;18(3):280-6.

7. Kuderer NM, Dale DC, Crawford J, Lyman GH. Impact of primary prophylaxis with granulocyte colonystimulating factor on febrile neutropenia and mortality in adult cancer patients receiving chemotherapy: a systematic review. J Clin Oncol 2007;25(21):3158-67.

8. Yang BB, Savin MA, Green M. Prevention of chemotherapy-induced neutropenia with pegfilgrastim: pharmacokinetics and patient outcomes. Chemotherapy 2012;58(5):387-98.

9. Green MD, Koelbl H, Baselga J, Galid A, Guillem V, Gascon P, et al. A randomized double-blind multicenter phase III study of fixed-dose single-administration pegfilgrastim versus daily. Ann Oncol 2003;14(1):2935.

10. Siena S, Piccart MJ, Holmes FA, Glaspy J, Hackett J, Renwick JJ. A combined analysis of two pivotal randomized trials of a single dose of pegfilgrastim per chemotherapy cycle and daily Filgrastim in patients with stage II-IV breast cancer. Oncol Rep 2003;10(3):715-24.

11. Vogel CL, Wojtukiewicz MZ, Carroll RR, Tjulandin SA, Barajas-Figueroa LJ, Wiens BL, et al. First and Subsequent Cycle Use of Pegfilgrastim Prevents Febrile Neutropenia in Patients With Breast Cancer: A Multicenter, Double-Blind, Placebo-Controlled Phase III Study. J Clin Oncol 2005 20;23(6):1178-84.

12. Holmes FA, O’Shaughnessy JA, Vukelja S, Jones SE, Shogan J, Savin M, et al. Blinded, randomized, multicenter study to evaluate single administration pegfilgrastim once per cycle versus daily filgrastim as an adjunct to chemotherapy in patients with high-risk stage II or stage III/IV breast cancer. J Clin Oncol 2002;20(3):727-31.

13.Zamboni WC. Pharmacokinetics of pegfilgrastim. Pharmacotherapy 2003;23(8 Pt 2):9S-14S.

14. Klastersky J, de Naurois J, Rolston K, Rapoport B, Maschmeyer G, Aapro M, et al. Management of febrile neutropaenia: ESMO Clinical Practice Guidelines. Ann Oncol 2016;27(suppl 5):v111-v8.

15. Salmon JP, Smakal M, Karanikiotis C, Wojtukiewicz MZ, Omnes Y, DeCosta L, et al. Febrile neutropenia (FN) and pegfilgrastim prophylaxis in breast cancer and non-Hodgkin's lymphoma patients receiving high (> 20\%) FN-risk chemotherapy: results from a 
prospective observational study. Support Care Cancer 2019;27(4):1449-57.

16. Eckstrom J, Bartels T, Abraham I, Patel H, Elquza E, Scott AJ, et al. A single-arm, retrospective analysis of the incidence of febrile neutropenia using sameday versus next-day pegfilgrastim in patients with gastrointestinal cancers treated with FOLFOX or FOLFIRI. Support Care Cancer 2019;27(3):873-8.
17. Pawloski PA, Larsen M, Thoresen A, Giordana MD. Pegfilgrastim use and bone pain: a cohort study of community-based cancer patients. J J Oncol Pharm Pract 2016;22(3):423-9.

18. Xu H, Gong Q, Vogl FD, Reiner M, Page JH. Risk Factors For Bone Pain Among Patients Receiving Myelosuppressive Chemotherapy and Primary Prophylactic Pegfilgrastim. Support Care Cancer. 2016;24(2):723-30. 